\title{
Precritical soft photon emission from quark matter
}

\author{
B. O. Kerbikov $\circledast^{*}$ \\ Lebedev Physical Institute, Moscow 119991, Russia \\ Moscow Institute of Physics and Technology, Dolgoprudny 141700, \\ Moscow Region, Russia
}

(Received 21 March 2020; revised 17 June 2020; accepted 13 October 2020; published 24 November 2020)

\begin{abstract}
We compute the soft real photon emission rate from the QCD matter in the vicinity of the critical line at moderate density and the temperature approaching the critical one from above. The obtained production rate exhibits a steep rise close to $T_{c}$ due to the formation of the slow fluctuation mode.
\end{abstract}

DOI: 10.1103/PhysRevD.102.096022

\section{INTRODUCTION}

Heavy ion collision experiments carried out at the Relativistic Heavy Ion Collider (RHIC) and LHC over the last two decades brought about the discovery of a new form of matter with unexpected properties. Several probes are used to reveal its nature and characteristics. A special role is played by direct photons. They are produced at all stages of the fireball evolution and can easily escape the collision region without reinteracting. Photon and dilepton production has been studied both experimentally and theoretically for quite a long time. The basic theory concepts have their roots in the studies performed several decades ago [1-5]. The current status of the field is presented in the review article of Ref. [6]. In this work, we consider the real soft photon emission rate from dense quark matter with the temperature approaching the critical one from above. Real photons means that $q^{2}=\omega^{2}-\mathbf{q}^{2}=M^{2}=0$, and soft corresponds to $\omega \ll T$. For the dilepton production, $M^{2}>0$ is the invariant mass of the lepton pair. This process is not considered in the present work. It is necessary to emphasize that only the external photon is assumed to be soft but the internal momenta in the self-energy diagram may be hard. In a sense, the picture is reminiscent of the hard thermal loop approximation. The role of high $T$ is played by the high chemical potential. The dominant contribution to the photon polarization operator comes from the vicinity of the Fermi surface. Up to now, the soft photon emission has been predominantly studied for hot and low-density QGP. In this region of the QCD phase diagram, perturbative methods including the hard thermal loop are the adequate

\footnotetext{
*borisk@itep.ru
}

Published by the American Physical Society under the terms of the Creative Commons Attribution 4.0 International license. Further distribution of this work must maintain attribution to the author(s) and the published article's title, journal citation, and DOI. Funded by SCOAP. research tools [7-11]. Results of several lattice calculations at zero chemical potential are also available [12-14]. On the other hand, during the last few years, it became clear that, except for the high-temperature and low-density domain, the quark matter is a strongly coupled medium [15]. There are very few calculations of the photon production beyond, or partly beyond, the perturbation theory [16-19].

The reason is that the finite-temperature retarded selfenergy of virtual photon is known only in perturbation theory $[20,21]$. Probably the most intriguing region of the phase diagram lies in the vicinity of the critical temperature at nonzero density. The corresponding research program is planned at NICA (Nuclotron Ion Collider Activity, Dubna, Russia) and FAIR (Facility for Antiproton and Ion Research, Darmstadt, Germany). In this domain, the correlation functions are characterized by the presence of a soft mode of the fluctuation field.

The importance of the collective mode in the precritical region of the quark matter at finite density and its relevance for the dilepton production was to our knowledge first pointed out in Refs. [22,23].

It will be shown below that the propagator of the fluctuation mode (FP) has the form

$$
L(\mathbf{q}, \omega)=\frac{N}{\frac{T-T_{c}}{T_{c}}-i \beta \omega+\xi^{2} \mathbf{q}^{2}} .
$$

The quantities $N, \beta$, and $\xi^{2}$ will be determined in what follows. One may recognize in (1) the linear response function of the phase transition theory [24,25]. At small $\omega$ and $\mathbf{q}^{2}$ and close to $T_{c}$, the FP (1) can be arbitrary large and is rapidly varying due to the $\left(T-T_{c}\right) / T_{c}$ term. We shall evaluate the soft photon emission rate close to $T_{c}$ using the expression for the retarded self-energy containing two FPs. This will lead to the enhanced soft photon production rate.

The organization of this paper is as follows. In Sec. II, we show that there is a rather wide fluctuation region above the critical line at moderate density. In Sec. III, using the time-dependent Ginzburg-Landau functional with 
Langevin forces, we derive the propagator of the soft collective mode. In Sec. IV, we address the retarded photon self-energy in the fluctuation region. In Sec. V, we compute the soft photon emissivity and confront it with the electrical conductivity computation. We summarize and conclude in Sec. VI.

\section{CRITICAL FLUCTUATIONS}

Our focus in this work is on the finite-density precritical fluctuation region with $T \rightarrow T_{c}$ from above. Comprehensive study has shown that at high density and low temperature the ground state of QCD is a color superconductor [26,27]. We consider the 2CS (two flavor color superconductor) color superconducting phase when $u$ and $d$ quarks participate in color antitriplet pairing but the density is not high enough to involve the heavier $s$ quark. The value of the quark chemical potential under consideration is $\mu \simeq 300-400 \mathrm{MeV}$, and the critical temperature is $T_{c} \simeq 40-50 \mathrm{MeV}$. The corresponding density is two or three times the normal nuclear density. Both numbers should be considered as an educated guess since they rely on model calculations. A similar choice of parameters has been adopted in Ref. [22], namely, $\mu \simeq 400-500 \mathrm{MeV}$ and $T_{c} \simeq 40-60 \mathrm{MeV}$. Prior to forming a condensate, the system goes through the phase of the preformed fluctuation quark pairs. In its basic features, this state is very different from the fluctuation regime of the BCS (Bardeen-CooperSchrieffer) superconductor [28]. In the BCS, the border between the normal and the superconducting phases is very sharp. In color superconductor, it is significantly smeared. Two interrelated explanations of this difference may be given. First, in the BCS, the characteristic pair correlation length $\xi$ is large, $\xi \simeq 10^{-4} \mathrm{~cm}$, so that $n_{e}^{1 / 3} \xi \gg 1$, where $n_{e} \sim 10^{22} \mathrm{~cm}^{-3}$ is the electron density [29]. The pairs strongly overlap. In color superconductor, the pairs which form the condensate are much more compact and have a small overlap (the Schafroth Pairs [30]). The role of the correlation length $\xi$ is taken by the root-mean-square radius $Q \sim 1 \mathrm{fm}$ of the quark pair. The $2 \mathrm{SC}$ quark matter density $n_{q}$ is two or three times the normal nuclear density so that $n_{q}^{1 / 3} Q \sim 1$. Note that $n^{1 / 3} \xi$ is the BCS-BEC (Bose-Einstein condensation) crossover parameter [31-36]. Therefore, one may say that at $\mu \sim 300-400 \mathrm{MeV}, T \sim 40-50 \mathrm{MeV}$ the system is in the crossover regime [28]. The second way to reveal the difference between the BCS and color superconductor is to compare the relative values of the energy parameters in the two theories. In the BCS, the scales hierarchy holds, $\Delta: \omega_{D}: \varepsilon_{F} \simeq 1: 10^{2}: 10^{4}$, where $\Delta \sim T_{c} \sim$ $10^{-4} \mathrm{eV}$ is the gap/critical temperature, $\omega_{D} \sim 10^{-2} \mathrm{eV}$ is the Debye energy, and $\varepsilon_{F} \sim 2 \mathrm{eV}$ is the Fermi energy [29]. In color superconductor, the relation is very different, $\Delta: \Lambda: \mu \simeq 1: 8: 4$, where $\Delta \sim 0.1 \mathrm{GeV}$ is the gap, $\Lambda \sim$ $0.8 \mathrm{GeV}$ is the UV cutoff, and $\mu \sim 0.4 \mathrm{GeV}$ is the quark chemical potential [35]. The width of the fluctuation region and the fluctuation contribution to the physical quantities are characterized by the Ginzburg-Levanyuk parameter [24,28,29,35,37-39]. There are several definitions of this quantity in the literature $[24,29,37]$. The underlying requirement is that the fluctuation corrections to the physical quantities (e.g., the heat capacity and the electrical conductivity) must be much smaller than the characteristic values of these quantities. In the BCS theory the estimate of the temperature interval dominated by fluctuations was first given by Ginzburg [39] It is based on the heat capacity contribution due to fluctuations and reads

$$
\mathrm{Gi} \simeq \frac{\delta T}{T_{c}} \sim\left(\frac{T_{c}}{E_{F}}\right)^{4}
$$

where $E_{F}$ is the Fermi energy. To adjust this estimate to the quark matter, we replace $E_{F}$ by $\mu$, use the BCS theory estimate $\xi \sim T_{c}^{-1}[29,37]$, and then replace $\xi$ by the quark pair radius $\varrho$. It should be noted that the rigorous calculation of the pair size in the nonperturbative QCD region is hardly possible. The energy spread of the correlated pair of quarks is $\delta E \sim \Delta \sim 100 \mathrm{MeV}$. Since the quarks are relativistic, their momentum spread is of the same order $\delta p \sim \Delta$. Therefore the pair size is $\varrho \sim 2 \mathrm{fm}$. Using the Klein-Gordon equation for the quark pair [40], one can obtain an estimate $Q \simeq(\sqrt{3} \Delta)^{-1} \sim 1 \mathrm{fm}$. Equation (2) describes the universal dependence of $\mathrm{Gi}$ on the superconductor physical parameters. Depending on the specific properties of a given material, it should be supplemented by an additional numerical factor [24,37]. The evaluation of this factor for the quark matter is a difficult problem. We shall not try to solve it since Eq. (2) contains a strong fourth power dependence on $T_{c}, \mu, Q$, and the overall numerical coefficient is less important. As we discussed above, the values of these parameters are not narrowly limited. Replacing in (2) $E_{F}$ by $\mu$ and using the estimate $\varrho \sim T_{c}^{-1}$, we write the following two complementary expressions for the Ginzburg parameter:

$$
\mathrm{Gi} \simeq \frac{\delta T}{T_{c}} \simeq\left(\frac{T_{c}}{\mu}\right)^{4} \simeq(\mu \varrho)^{-4} .
$$

Because of the fourth power dependence on $T_{c}, \mu$, and $\varrho$ and because of some uncertainty in their values, we can estimate only the reliable interval of the Gi parameter. For $T_{c} \simeq(40-50) \mathrm{MeV}, \mu \simeq(300-400) \mathrm{MeV}, \varrho \simeq(1-2) \mathrm{fm}$, the quantity $\mathrm{Gi}$ varies from $10^{-4}$ to $10^{-2}$. We remind the reader that for the ordinary superconductors $\mathrm{Gi} \sim 10^{-14}$ $10^{-12}[29,37]$. In the next section, we shall discuss the bound on Gi from below.

\section{COLLECTIVE MODE PROPAGATOR}

The FP of the form (1) may be derived in several ways. In Ref. [41], it was obtained by solving the Dyson equation with relativistic Matsubara quark propagators. Here, we 
shall use the time-dependent Ginzburg-Landau (GL) functional $[42,43]$ with the stochastic Langevin forces. The approximations and omissions in the derivation to follow will be discussed at the end of this section. In absence of the external electromagnetic field, the time-dependent GL equation for the fluctuating pair field $\Psi(\mathbf{r}, t)$ reads

$$
-\gamma \frac{\partial}{\partial t} \Psi(\mathbf{r}, t)=\frac{\delta F[\Psi]}{\delta \Psi^{*}}+\eta(\mathbf{r}, t)
$$

Here, $\gamma$ is the order parameter relaxation constant, and $\eta(\mathbf{r}, t)$ are the Langevin forces. The GL functional with the quartic term dropped (see below) has the form [24,29,37]

$$
F[\Psi]=\nu \int\left[\varepsilon|\Psi(\mathbf{r}, t)|^{2}+\xi^{2}|\nabla \Psi(\mathbf{r}, t)|^{2}\right] d V d t,
$$

where $\nu=\mu p_{F} / \pi^{2}$ is the relativistic density of states at the Fermi surface $[28,41], \varepsilon=\left(T-T_{c}\right) / T_{c}$, and $\xi$ is the coherence length which may be expressed in terms of the diffusion coefficient as $\xi^{2}=\frac{\pi}{8 T} D[37,41]$. Referring the readers to the above references, we present a sketch of the derivation. The starting point is the QCD partition function. Expanding it in powers of $|\Psi|^{2}$, one arrives at the needed GL expression. The term $\xi^{2}|\nabla \Psi|^{2}$ in (5) enters into this expression with the coefficient equal to [28]

$$
\xi^{2}=\frac{7 \zeta(3) v_{F}^{2}}{48 \pi^{2} T^{2}} \chi\left(\frac{1}{2 \pi T \tau}\right)
$$

Here, $\tau$ is the momentum relaxation time. The function $\chi(z)$ is

$$
\begin{aligned}
\chi(z) & =\frac{8}{7 \zeta(3)} \sum_{n=0}^{\infty} \frac{1}{(2 n+1)^{2}(2 n+1+z)} \\
& \rightarrow \begin{cases}1, & z \rightarrow 0 ; \\
\frac{\pi^{2}}{7 \zeta(3)} z^{-1}, & z \gg 1 .\end{cases}
\end{aligned}
$$

The relaxation time $\tau$ depends on the temperature, density, and quark flavor. It can be also identified with the mean free path time, or the relaxation time in the Boltzmann approximation. The reliable estimation of $\tau$ is absent even for $\mu=0$. For example, in Ref. [44], it varies at $\mu=0$ in the interval $\tau \simeq(0.1-0.9) \mathrm{fm}$. Therefore, let us consider the two limiting cases, namely, $2 \pi T \tau \ll 1$ and $2 \pi T \tau \gg 1$. For the critical temperature under consideration, $T_{c} \simeq(40-50) \mathrm{MeV}$, the two limits take place at $\tau \lesssim$ $0.3 \mathrm{fm}$ and $\tau \gtrsim 2 \mathrm{fm}$ correspondingly. Based on our experience in the calculation of the quark matter conductivity [41], we consider the choice $\tau \lesssim 0.3 \mathrm{fm}$ more realistic. In the above two limits, one obtains correspondingly

$$
\xi^{2} \simeq \frac{\pi}{8 T}\left(\frac{1}{3} v_{F}^{2} \tau\right) \equiv \frac{\pi}{8 T} D_{1}
$$

$$
\xi^{2} \simeq \frac{\pi}{8 T}\left(\frac{v_{F}^{2}}{6 \pi T}\right) \equiv \frac{\pi}{8 T} D_{2}
$$

The quantity $D_{1}$ is a standard diffusion coefficient $D_{1} \sim v l$. The coefficient $D_{2}$ has a meaning of a diffusion coefficient in the quasifree ballistic regime [37]. It can be obtained from (8) by the replacement $\tau \rightarrow(2 \pi T)^{-1}$. We consider a rather dense quark matter. It is in a collisional "dirty" regime, not in a ballistic one. Therefore, in our calculations, we shall take $\xi^{2}$ in the form (8), omit the lower subscript, and slightly vary the parameter $\tau$.

Now, we perform a Fourier transform to momentum space,

$$
\Psi(\mathbf{r}, t)=\int \frac{d \mathbf{q}}{(2 \pi)^{3}} \frac{d \omega}{2 \pi} e^{i \mathbf{q r}-i \omega t} \phi(\mathbf{q}, \omega) .
$$

The GL functional in momentum space reads

$F[\phi]=\nu \int \frac{d \mathbf{q}}{(2 \pi)^{3}} \frac{d \omega}{2 \pi}\left[\left(\varepsilon+\frac{\pi}{8 T} D q^{2}\right)|\phi(\mathbf{q}, \omega)|^{2}\right]$.

The time-dependent Eq. (4) takes the following form in momentum space:

$$
-\left[-i \gamma \omega+\nu\left(\varepsilon+\frac{\pi}{8 T} D \mathbf{q}^{2}\right)\right] \phi(\mathbf{q}, \omega)=\eta(\mathbf{q}, \omega) .
$$

The solution of (12) may be written as

$$
\phi(\mathbf{q}, \omega)=L(\mathbf{q}, \omega) \eta(\mathbf{q}, \omega),
$$

where

$$
L(\mathbf{q}, \omega)=-\left(-i \gamma \omega+\Omega_{\mathbf{q}}\right)^{-1}
$$

with $\Omega_{\mathbf{q}}=\nu\left(\varepsilon+\frac{\pi}{8 T} D q^{2}\right)$. To ascertain that $L$ is actually the fluctuation mode propagator, we must verify that it satisfies the fluctuation-dissipation theorem $[24,45]$. The theorem states that the equal time correlator $\left\langle\Psi(\mathbf{r}, t) \Psi^{*}\left(\mathbf{r}^{\prime}, t\right)\right\rangle$ is expressed via the retarded propagator. The solution (13) satisfies this requirement, provided the correlator of the Langevin forces have a Gaussian white noise form in the coordinate space

$$
\left\langle\eta(\mathbf{r}, t) \eta^{*}\left(\mathbf{r}^{\prime}, t^{\prime}\right)\right\rangle=2 T \gamma \delta\left(\mathbf{r}-\mathbf{r}^{\prime}\right) \delta\left(t-t^{\prime}\right) .
$$

Then,

$$
\left\langle\eta(\mathbf{r}, t) \eta^{*}\left(\mathbf{r}^{\prime}, t\right)\right\rangle=2 T \gamma \int \frac{d \mathbf{q}}{(2 \pi)^{3}} e^{-i \mathbf{q}\left(\mathbf{r}-\mathbf{r}^{\prime}\right)} \int \frac{d \omega}{2 \pi},
$$

and 


$$
\begin{aligned}
\left\langle\Psi(\mathbf{r}, t) \Psi^{*}\left(\mathbf{r}^{\prime}, t\right)\right\rangle= & 2 T \gamma \int \frac{d \mathbf{q}}{(2 \pi)^{3}} e^{i \mathbf{q}\left(\mathbf{r}-\mathbf{r}^{\prime}\right)} \\
& \times \int_{-\infty}^{\infty} \frac{d \omega}{2 \pi} L(\mathbf{q}, \omega) L^{*}(\mathbf{q}, \omega) .
\end{aligned}
$$

Therefore, $\left\langle\Psi(\mathbf{r}, t) \Psi^{*}\left(\mathbf{r}^{\prime}, t\right)\right\rangle$ in momentum space is

$$
\begin{aligned}
\left\langle\Psi(\mathbf{r}, t) \Psi^{*}\left(\mathbf{r}^{\prime}, t\right)\right\rangle_{\mathbf{p}} & =2 T \gamma \int_{-\infty}^{\infty} \frac{d \omega}{2 \pi} L(\mathbf{q}, \omega) L^{*}(\mathbf{q}, \omega) \\
& =-\int \frac{d \omega}{2 \pi} \frac{2 T}{\omega} \operatorname{Im} L(\mathbf{q}, \omega) .
\end{aligned}
$$

Thus, $L(\mathbf{q}, \omega)$ given by (14) meets the needed requirement. The last step is to express the coefficient $\gamma$ in terms of other parameters. From (12), it follows that the relaxation time of fluctuations with momentum $\mathbf{q}^{2}$ is

$$
\tau=\frac{\gamma}{\nu\left(\varepsilon+\frac{\pi}{8 T} D \mathbf{q}^{2}\right)} .
$$

Keeping in the denominator of (19) only the term $\nu \varepsilon$, which is equivalent to retaining only the term $\varepsilon|\psi|^{2}$ in (5), and comparing the result with the GL decay time $\tau_{G L}=$ $\pi\left[8\left(T-T_{c}\right)\right]^{-1}[29,37]$, we obtain $\gamma=\pi \nu / 8 T_{c}$. This completes the derivation of the FP:

$$
L(\mathbf{q}, \omega)=-\frac{1}{\nu\left[\varepsilon+\frac{\pi}{8 T}\left(-i \omega+D \mathbf{q}^{2}\right)\right]} .
$$

We note that, since we consider the temperature interval $\varepsilon \lesssim 10^{-2}$, the temperature $T$ in (20) may be replaced by $T_{c}$ without a noticeable loss of accuracy. We do not find this simplification necessary. The way the above results were obtained may raise questions about the validity of the employed approximations.

Let us discuss the debatable points. The electromagnetic field was not included in Eq. (5). It is well known that the external magnetic field applied to the superconductor gives rise to important phenomena like the Meissner effect. It also influences the physics of fluctuations in ordinary superconductors [37] as well as in color superconductors [28]. Quark matter may be embedded into magnetic field when it is produced in the peripheral collisions of the ultrarelativistic heavy ions collisions at the RHIC and LHC [46]. Quark-gluon matter formed in such collisions has high temperature and low density, which excludes the formation of the color quark confinement. The present investigation may be important for the future experiments at NICA and FAIR where the sizable magnetic field, if any, will not be generated. The omission of the fourth-order term in (5) is a subtle question. Without this term, Eq. (5) corresponds to the Gaussian fluctuations with no interaction between them. In the immediate vicinity of $T_{c}$ at $\varepsilon \lesssim \mathrm{Gi}$, this approximation breaks down [24,29,37]. Here, one encounters a difficult problem. The renormalization group method is used in this critical region [24,37]. However, in the threedimensional case, the complete solution is lacking, and we shall not dwell on that. Based on the values of Gi obtained in the previous section, we shall present the results down to $\varepsilon \simeq 10^{-4}$, keeping in mind that below $\varepsilon \simeq 10^{-2}$ the corrections due to the interaction between fluctuations may come into play.

\section{PHOTON PRECRITICAL SELF-ENERGY}

To calculate the photon emission rate, we have to construct the photon self-energy operator in the precritical region. Intensive studies since the 1960s have resulted in a fairly complete picture of the fluctuation effects near $T_{c}$ see Ref. [37] and a long list of references therein. Three basic papers [47-49] should be singled out of this list. Worth mentioning also Ref. [50], in which the fluctuation conductivity has been studied in the strong coupling limit. The quark pairs under study in this work are in the strong coupling regime close to the BCS-BEC crossover [28,36].

According to the diagram calculus, the self-energy $\Pi(\mathbf{q}, \omega)$ in the precritical region can be constructed from the two kinds of the building blocks. These are the quark Matsubara Green's functions $G\left(\mathbf{p}, \varepsilon_{n}\right)$ (see below) and the fluctuating field pair average represented by the FP (20). The GL functional (5) without the fourth-order term describes an almost free field. For the free field, the Wick theorem states that the higher-order correlators are expressed as products of the pair averages, i.e., the FPs. Therefore, we are really left with the two above building blocks. Attributing the solid lines to the quark propagators and the wavy lines to the FPs, we come to the set of diagrams for the photon self-energy (the retarded Green's function). The possible diagrams have been discussed in a vast number of works; see Ref. [37] for the review and Refs. [47-49] for the original results. The two diagrams which were compared in a number of publications are the Aslamazov-Larkin [47] and the Maki-Thompson [48,49] ones. It is beyond the scope of this paper to reproduce their comparative analysis $[29,37,51]$. The bottom line is that the theoretical arguments supported by the experimental data [52] allow us to conclude that the dominant role is played by the celebrated Aslamazov-Larkin (AL) diagram [37,47,51] shown in Fig. 1.

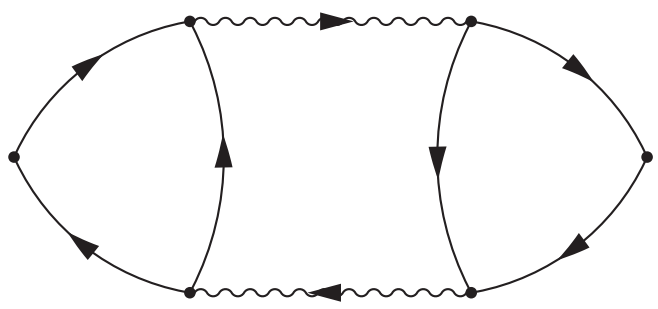

FIG. 1. The AL diagram for the polarization operator. The solid lines correspond to the Matsubara quark Green's functions, and the wavy lines correspond to the FPs. 
It consists of two quark loops connected by FPs and reads

$$
\begin{aligned}
& \Pi_{l m}\left(\omega=i \omega_{k}=|\mathbf{k}|\right) \\
& =-3 Q^{2} T \sum_{\Omega j} \int \frac{d \mathbf{q}}{(2 \pi)^{3}} B_{l}\left(\mathbf{k}, \mathbf{q}, \Omega_{j}, \omega_{k}\right) \\
& \quad \times L\left(\mathbf{k}+\mathbf{q}, \Omega_{j}+\omega_{k}\right) B_{m}\left(\mathbf{k}, \mathbf{q}, \Omega_{j}, \omega_{k}\right) L\left(\mathbf{q}, \Omega_{j}\right) .
\end{aligned}
$$

Here, $\omega_{k}$ and $\Omega_{j}$ are the Matsubara frequencies. The prefactor 3 in (21) comes from three colors of quarks, $Q^{2}=$ $\frac{5}{9} e^{2}$ for two flavors, $e^{2}=4 \pi \alpha$. The trace over the Dirac indices is included in the 3-vector $\mathbf{B}$ with components $B_{l}$ and $B_{m}$. The factor $\mathbf{B}$ corresponds to the three Green's functions block. Two points concerning Eq. (21) deserve an explanation. The first one is that the dependence of the quark loop $\mathbf{B}$ on $\mathbf{k}$ can be dropped out and therefore the self-energy $\Pi$ is a function of $\omega=|\mathbf{k}|$ as it should be for real photons. Second, the 3 -vector $\mathbf{B}$ is by symmetry arguments proportional to $\mathbf{q}, \mathbf{B} \sim \mathbf{q}$. The quark loop $\mathbf{B}$ is given by the following expression:

$$
\begin{aligned}
& \mathbf{B}\left(\mathbf{k}, \mathbf{q}, \Omega_{j}, \omega_{k}\right) \\
& =T \sum_{\varepsilon_{n}} \lambda\left(\mathbf{q}, \varepsilon_{n}+\omega_{k}, \Omega_{j}-\varepsilon_{n}\right) \lambda\left(\mathbf{q}, \varepsilon_{n}, \Omega_{j}-\varepsilon_{n}\right) \\
& \quad \cdot \int \frac{d \mathbf{p}}{(2 \pi)^{3}} \operatorname{tr}_{D}\left[\vec{\gamma} G\left(\mathbf{p}, \tilde{\varepsilon}_{n}\right) G\left(\mathbf{p}+\mathbf{k}, \tilde{\varepsilon}_{n}+\omega_{k}\right)\right. \\
& \left.\quad \times G\left(\mathbf{q}-\mathbf{p}, \Omega_{j}-\tilde{\varepsilon}_{n}\right)\right] .
\end{aligned}
$$

The Matsubara propagators in (21) have the form

$$
G\left(\mathbf{p}, \tilde{\varepsilon}_{n}\right)=\frac{1}{\gamma_{0}\left(i \tilde{\varepsilon}_{n}+\mu\right)-\vec{\gamma} \mathbf{p}-m},
$$

where $\tilde{\varepsilon}_{n}=\varepsilon_{n}+\frac{1}{2 \tau} \operatorname{sgn} \varepsilon_{n}, \varepsilon_{n}=\pi T(2 n+1)$, where $\tau$ is the momentum relaxation time. This quantity was already introduced in Sec. III. Alternatively, $\tau$ may be called the mean free path time. It enters into the Drude formula for the quark matter conductivity and into the Boltzmann equation in the relaxation time approximation [41]. From the formal point of view, $\tau$ regulates the pinch (collinear) singularities. The factors $\lambda$ are the vertex renormalization corrections [37,51]. At $\mathbf{q} \rightarrow 0, \omega_{k} \rightarrow 0$, the product of the two $\lambda$ s takes the limiting value $\left|2 \tilde{\varepsilon}_{n}\right|^{2} /\left|\varepsilon_{n}\right|^{2}$ [41,51]. The quark loop (22) is calculated under the following conditions: (i) $|\mathbf{k}| \ll|\mathbf{p}|$ and (ii) $T \ll \mu$. The first condition is easily recognized as the hard thermal loops approximation (HTL). The external momentum $\mathbf{k}$ is assumed to be soft since we are interested in the soft photon emission, but internal momentum $\mathbf{p}$ is hard. However, in our case, this is not due to the high temperature as in the standard HTL but due to the fact that the dominant contribution to the above integral comes from the vicinity of the Fermi surface with $\mathbf{p} \sim \mu$, and $\mu \simeq 300-400 \mathrm{MeV}$, i.e., high. Therefore, we replace in (22)
$G\left(\mathbf{p}+\mathbf{k}, \tilde{\varepsilon}_{n}+\omega_{k}\right) \simeq G\left(\mathbf{p}, \tilde{\varepsilon}_{n}+\omega_{k}\right)$, and $\mathbf{B}$ becomes $\mathbf{k}$ independent. By symmetry arguments, $\mathbf{B} \sim \mathbf{q}$. Integration in (22) is performed using the Fermi surface integration measure

$$
\int \frac{d \mathbf{p}}{(2 \pi)^{3}}=\frac{\nu}{2} \int \frac{d \Omega_{\mathbf{p}}}{4 \pi} \int_{-\infty}^{\infty} d t
$$

where $t=\sqrt{\mathbf{p}^{2}+m^{2}}-\mu, \nu=\frac{\mu p_{F}}{\pi^{2}}$. In the vicinity of $T_{c}$, the FP (20) has a pole structure due to the $\varepsilon$ term. The dependence of $L\left(\mathbf{q}, \Omega_{j}\right)$ and $L\left(\mathbf{q}, \Omega_{j}-\omega_{k}\right)$ on $\Omega_{j}$ and $\omega_{k}$ is much stronger than the dependence of the Green's functions on the same quantities. We shall keep in the propagators entering into $\mathbf{B}\left(\mathbf{q}, \Omega_{j}, \omega_{k}\right)$ only the dependence on the fermionic frequencies $\tilde{\varepsilon}_{n}$ and evaluate $\mathbf{B}\left(\mathbf{q}, \Omega_{j}=\omega_{k}=0\right)$. Expanding $G\left(\mathbf{q}-\mathbf{p},-\tilde{\varepsilon}_{n}\right)$ in (22) at $\mathbf{q} \rightarrow 0$, one has

$$
\begin{aligned}
G\left(\mathbf{q}-\mathbf{p},-\tilde{\varepsilon}_{n}\right) & \simeq G\left(-\mathbf{p},-\tilde{\varepsilon}_{n}\right)+\mathbf{q} \frac{\partial}{\partial \mathbf{p}} G\left(-\mathbf{p},-\tilde{\varepsilon}_{n}\right) \\
& =G\left(-\mathbf{p},-\tilde{\varepsilon}_{n}\right)+\frac{(\mathbf{q p})}{\mu} \frac{\partial}{\partial t} G\left(-\mathbf{p},-\tilde{\varepsilon}_{n}\right) .
\end{aligned}
$$

Substituting (25) in (22), one easily observes that the angular integration kills the contribution of the first term of (25). The second term yields

$$
\mathbf{B}(\mathbf{q})=-\nu T \sum_{\varepsilon_{n}} \frac{\left|2 \tilde{\varepsilon}_{n}\right|^{2}}{\left|\varepsilon_{n}\right|^{2}} \int \frac{d \Omega_{\mathbf{p}}}{4 \pi} \frac{(\mathbf{q p}) \mathbf{p}}{\mu^{2}} \int_{-\infty}^{\infty} \frac{d y}{\left(y^{2}+\tilde{\varepsilon}_{n}^{2}\right)^{2}} .
$$

Performing the integration and using (7)-(9), one gets

$$
\mathbf{B}(\mathbf{q})=-\mathbf{q} \frac{7 \zeta(3)}{12} \frac{\nu}{\pi^{2} T^{2}} \frac{\mathbf{p}^{2}}{\mu^{2}} \chi\left(\frac{1}{2 \pi T \tau}\right)=-4 \mathbf{q} \frac{\pi \nu}{8 T} D,
$$

where $D$ are the diffusion coefficients defined by (8). From (21) and (27), we have

$$
\begin{aligned}
\Pi_{l m}\left(\omega_{k}\right)= & -12 Q^{2} T\left(\frac{\pi \nu}{8 T}\right)^{2} D^{2} \sum_{\Omega_{j}} \int \frac{d \mathbf{q}}{(2 \pi)^{3}} q_{l} q_{m} L\left(\mathbf{q}, \Omega_{j}\right) \\
& \times L\left(\mathbf{q}, \Omega_{j}+\omega_{k}\right) .
\end{aligned}
$$

To evaluate the sum in (28), we can use a technique of replacing the summation (28) by the contour integration (the so-called Eliashberg trick) [37,53]

$$
T \sum_{\Omega_{j}} f\left(\Omega_{j}\right)=\frac{1}{4 \pi i} \oint d z \operatorname{coth} \frac{z}{2 T} f(-i z),
$$

where $z=i \Omega_{j}$. The contour of integration is depicted in the original work [53] and in Ref. [37]. In (28), the FPs are 
defined over the discrete bosonic Matsubara frequencies. We have to perform the analytic continuation of the FPs. The retarded one, $L^{R}(\mathbf{q},-i z)$, is analytic in the upper half-plane $\operatorname{Im} z>0$, and the advanced one, $L^{A}(\mathbf{q},-i z)$, does not have singularities in the lower half-plane. Note that the FP given by (20) is the $L^{R}$ one. The $L^{A}$ is obtained by replacing in (20) $\omega \rightarrow-\omega$. Performing the contour integration [37,53], one gets

$$
\begin{aligned}
\Pi(\omega)= & -\frac{\pi Q^{2} \nu^{2} D^{2}}{32 T^{2}} \int \frac{d \mathbf{q}}{(2 \pi)^{3}} \mathbf{q}^{2} \int_{-\infty}^{\infty} d z \operatorname{coth} \frac{z}{2 T} \\
& \times\left[L^{R}(\mathbf{q},-i z-i \omega)+L^{A}(\mathbf{q},-i z+i \omega)\right] \\
& \times \operatorname{Im} L^{R}(\mathbf{q},-i z) .
\end{aligned}
$$

Next, we expand the integrand in powers of $\omega$ and subtract the zeroth-order term. This may be regarded as imposing the Ward identity. The term linear in $\omega$ reads

$$
\begin{aligned}
& L^{R}(\mathbf{q},-i z-i \omega)+L^{A}(\mathbf{q},-i z+i \omega) \\
& =-\omega \frac{d}{d z}\left(L^{R}(\mathbf{q},-i z)-L^{A}(\mathbf{q},-i z)\right) \\
& =-2 i \omega \frac{d}{d z} \operatorname{Im} L^{R}(\mathbf{q},-i z) .
\end{aligned}
$$

Substituting (31) into (30) and integrating by parts, we obtain

$\Pi(\omega)=-i \omega \frac{\pi Q^{2} \nu^{2} D^{2}}{32 T^{3}} \int \frac{d \mathbf{q}}{(2 \pi)^{3}} \mathbf{q}^{2} \int_{-\infty}^{\infty} d z \frac{\left[\operatorname{Im} L^{R}(\mathbf{q},-i z)\right]^{2}}{\operatorname{sh}^{2} \frac{z}{4 T}}$.

Expanding $\operatorname{sh}^{2} \frac{z}{4 T}$ at $z \ll 4 T$ and integrating over $d z$, we obtain

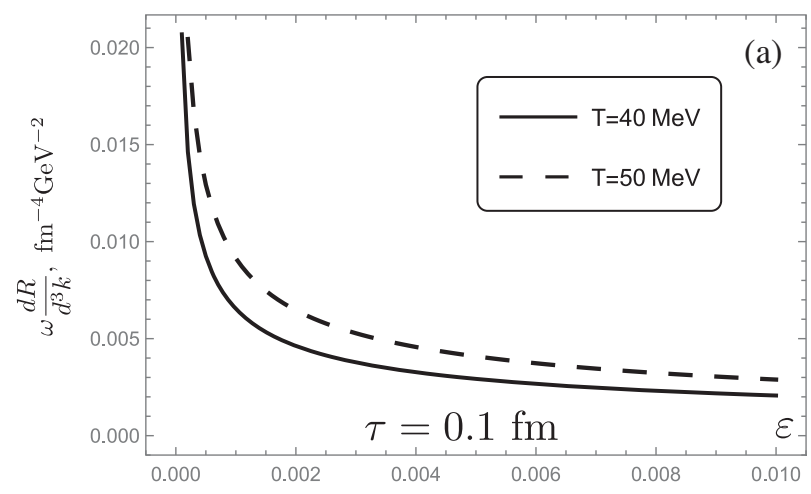

$$
\begin{aligned}
\Pi(\omega) & =-i \omega \frac{\pi^{3} Q^{2} D^{2}}{128 T^{2}} \int \frac{d \mathbf{q}}{(2 \pi)^{3}} \frac{\mathbf{q}^{2}}{\left(\varepsilon+\frac{\pi}{8 T} D \mathbf{q}^{2}\right)^{3}} \\
& =-i \omega \frac{3 Q^{2}}{64}\left(\frac{8 T}{\pi D}\right)^{1 / 2} \varepsilon^{-1 / 2} .
\end{aligned}
$$

As expected, the polarization operator is a singular function at $T \rightarrow T_{c}$ with the $\left(\frac{T}{T-T_{c}}\right)^{1 / 2}$ singularity.

\section{PHOTON EMISSION RATE}

The thermal emission rate of soft photons with energy $\omega$ is related to the retarded photon self-energy as [54]

$$
\omega \frac{d R}{d^{3} k}=-\frac{2}{(2 \pi)^{3}} \operatorname{Im} \Pi(\omega) \frac{1}{e^{\omega / T}-1} .
$$

Here, $\Pi(\omega)$ is the transverse projection of $\Pi_{\mu}^{\mu}$, and the longitudinal projection vanishes at $\mathbf{k}=0$. Using (33) for $\Pi(\omega)$, we obtain

$$
\omega \frac{d R}{d^{3} k}=\frac{3 Q^{2} T}{2^{8} \pi^{3}}\left(\frac{8 T}{\pi D}\right)^{1 / 2} \varepsilon^{-1 / 2} .
$$

Equation (34) is valid to order $e^{2}$ in electromagnetic interaction and to all orders in strong interaction. Expression (35) corresponds to the diagram shown in Fig. 1. It describes the emission of soft real photons with $\omega \ll T$ and is applicable within the precritical region $10^{-4}<\delta T / T_{c} \ll 1$. As was explained in Sec. III, corrections due to nonlinearity of fluctuations may come into play at $\delta T / T_{c} \simeq 10^{-4}$. In Fig. 2, the photon production rate is plotted as a function of $\varepsilon$ for $T_{c}=40 \mathrm{MeV}$ and $T_{c}=$ $50 \mathrm{MeV}$ and $\tau=0.1 \mathrm{fm}$ and $\tau=0.3 \mathrm{fm}$. The main feature of the emission rate (35) is its steep rise approaching $T_{c}$ from above. The dependence on $\tau$ is rather weak and on $T_{c}$ is not very pronounced.

As we mentioned in the Introduction, there are very few calculations of the photon emissivity at finite density. There are some common points between our results and that of

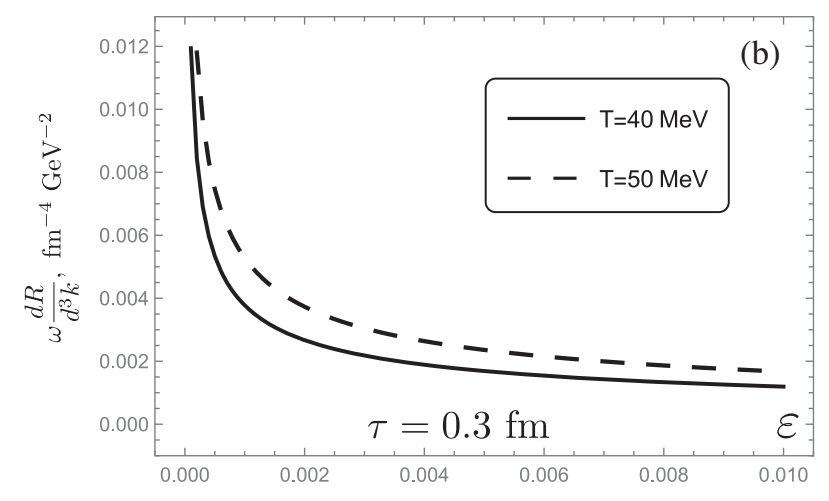

FIG. 2. Precritical soft photon emission rate. Panel (a): $\tau=0.1 \mathrm{fm}$; panel (b): $\tau=0.3 \mathrm{fm}$. The solid lines in both panels represent $T_{c}=40 \mathrm{MeV}$, and the dotted lines represent $T_{c}=50 \mathrm{MeV}$. 
Ref. [16]. The difference is that in Ref. [16] the quark matter is supposed to be in a color superconducting color flavor locked phase with quarks of three flavors $u, d$, and $s$ participating in pairing. In this work, we consider the precursor virtual pairing of $u$ and $d$ quarks at the temperature just above the critical one for the formation of the condensate. The bird's-eye view is that in Ref. [16] the characteristic soft photon emission rate is around $10^{-4} \mathrm{fm}^{-4} \mathrm{GeV}^{-2}$ (see Fig. 12 of Ref. [16]), while in our work, it is approximately $10^{-3} \mathrm{fm}^{-4} \mathrm{GeV}^{-2}$. It means that the slow fluctuation mode present in our study enhances the photon emissivity.

The soft photon radiation is closely related to the electrical conductivity of quark matter [12-14,55,56]. One can write the following equation for the electric current [57]:

$$
\mathbf{j}(x)=-\int \Pi(x-y) \mathbf{A}(y) d^{4} y .
$$

Replacing in Fourier transform of (36) $\mathbf{A}(\mathbf{k}, \omega)=$ $\mathbf{E}(\mathbf{k}, \omega) / i \omega$ and comparing with $\mathbf{j}=\sigma \mathbf{E}$, we obtain $[37,57]$

$$
\sigma(\omega)=-\frac{1}{\omega} \operatorname{Im} \Pi(\omega) .
$$

Comparison of (35) and (37) gives

$$
\omega \frac{d R}{d^{3} k}=\frac{2}{(2 \pi)^{3}} T \sigma(\omega) .
$$

Note that $\sigma(\omega)$ is of the same order $\alpha$ in electromagnetic interaction as the photon emissivity $\omega d R / d^{3} k$. The appearance of an additional factor $\alpha$ in the right-hand side of Eq. (II.16) of Ref. [13], Eq. (7) of Ref. [55], and Eq. (25) of Ref. [14] is unclear to the present author. Possibly, this is some problem of notations. One finds a large number of the quark matter electrical conductivity calculations in the literature; see, e.g., Ref. [41] and references therein.
Equations (35) and (38) yield for $\sigma$ at $T=0.05 \mathrm{GeV}$, $\tau=0.2 \mathrm{fm}$, and $\varepsilon^{-1 / 2}=20$ the result $\sigma=0.09 \mathrm{fm}^{-1}$. This value was previously obtained in our paper [41] dedicated to the electrical conductivity of quark matter.

\section{CONCLUSIONS}

In this paper, we have investigated the soft photon emission rate from dense quark matter in the precritical region. This region of the QCD phase diagram is not up to now thoughtfully investigated both experimentally and theoretically. We pursued the approach based on the Aslamazov-Larkin diagram, which proved to be very successful in condensed matter theory. For quark matter, this attitude allowed us to describe the transport anomalies near the phase transition temperature [58,59]. In particular, the bulk viscosity diverges near $T_{c}$ as $\zeta \sim \varepsilon^{-3 / 2}$ [58]. This is close to the critical behavior $\zeta \sim \varepsilon^{-z \nu+\alpha}, z \simeq 3, \nu \simeq 0.6$, $\alpha=0.11$ predicted in $d=4-\varepsilon$ renormalization, modes coupling, or isomorphism between the quark fluid and three-dimensional Ising system [60-63].

The most important feature of the soft photon emissivity rate is its rise when the temperature approaches $T_{c}$ from above. Close to $T_{c}$, the fluctuation radiation rate exceeds by an order of magnitude the rate from the color superconducting rate [16]. The origin of this phenomenon is the formation of the slow fluctuation made in the quark matter. This excitation is described by the fluctuation propagator, which is singular at $T_{c}$ in the limit $\omega \rightarrow 0$, $\mathbf{k} \rightarrow 0$. The enhancement of the soft photon production near $T_{c}$ may be a tentative proposal for the NICA/FAIR investigation.

\section{ACKNOWLEDGMENTS}

I thank Andrey Varlamov for illuminating discussions and Mikhail Lukashov for useful comments. I am grateful to Professor Masakiyo Kitazawa and Professor Teiji Kunihiro for pointing me to Refs. [22,23]. This work was supported by the RFBR Grant No. 18-02-40054.
[1] E. Shuryak, Sov. J. Nucl. Phys. 28, 408 (1978); Phys. Lett. 78B, 150 (1978).

[2] H. A. Weldon, Phys. Rev. D 28, 2007 (1983); 31, 545 (1985).

[3] K. Kajantie and H. I. Miettinen, Z. Phys. C 9, 341 (1981); 14, 357 (1982).

[4] L. D. McLerran and T. Toimela, Phys. Rev. D 31, 545 (1985).

[5] C. Gale and J. I. Kapusta, Nucl. Phys. B357, 65 (1991).

[6] G. David, Rep. Prog. Phys. 83, 046301 (2020).
[7] P. B. Arnold, G. D. Moore, and L. G. Yaffe, J. High Energy Phys. 11 (2001) 057.

[8] H. Gervais and S. Jeon, Phys. Rev. C 86, 034904 (2012).

[9] J. Ghiglieri, J. Hong, A. Kurkela, E. Lu, G. D. Moore, and D. Teaney, J. High Energy Phys. 05 (2013) 010.

[10] B. Zakharov, JETP Lett. 80, 1 (2004).

[11] E. Braaten, R. D. Pisarski, and T.-C. Yuan, Phys. Rev. Lett. 64, 2242 (1990).

[12] S. Gupta, Phys. Lett. B 597, 57 (2004). 
[13] H.-T. Ding, A. Francis, O. Kaczmarek, F. Karsch, E. Laermann, and W. Soeldner, Phys. Rev. D 83, 034504 (2011).

[14] H.-T. Ding, O. Kaczmarek, and F. Meyer, Phys. Rev. D 94, 034504 (2016).

[15] W. Busza, K. Rajagopal, and W. van der Schee, Annu. Rev. Nucl. Part. Sci. 68, 339 (2018).

[16] P. Jaikumar, R. Rapp, and I. Zahed, Phys. Rev. C 65, 055205 (2002).

[17] B. O. Kerbikov, 2019 QCD and high energy interactions, in Proceeding of the 54th Rencontres de Moriond QCD, ARISF (2019), p. 241.

[18] Y. Hidaka, Shu Lin, R. D. Pisarski, and D. Satow, J. High Energy Phys. 10 (2015) 005.

[19] C. A. Islam, S. Majumder, N. Haque, and M. G. Mustafa, J. High Energy Phys. 02 (2015) 011.

[20] M. Le Bellac, Thermal Field Theory (Cambridge University Press, Cambridge, England, 1996).

[21] J. I. Kapusta and C. Gale, Finite-Temperature Field Theory: Principles and Applications (Cambridge University Press, Cambridge, England, 2006).

[22] M. Kitazawa, T. Koide, T. Kunihiro, and Y. Nemoto, Prog. Theor. Phys. 114, 117 (2005).

[23] T. Kunihiro, M. Kitazawa, and Y. Nemoto, Proc. Sci. CPOD07 (2007) 041 [arXiv:0711.4429].

[24] A. Z. Patashinskii and V. L. Pokrovskii, Fluctuation Theory of Phase Transitions (Pergamon Press, Oxford, 1979).

[25] P. C. Hohenberg and B. I. Halperin, Rev. Mod. Phys. 49, 435 (1977).

[26] M. G. Alford, A. Schmitt, K. Rajagopal, and T. Schafer, Rev. Mod. Phys. 80, 1455 (2008).

[27] M. Buballa, Phys. Rep. 407, 205 (2005).

[28] B. O. Kerbikov and E. V. Lushevskaya, Phys. At. Nucl. 71, 364 (2008).

[29] V. V. Schmidt, The Physics of Superconductors, 2nd ed. (McGraw Hill, New York, 1974).

[30] M. R. Schafroth, Phys. Rev. 96, 1442 (1954).

[31] D. M. Eagles, Phys. Rev. 186, 456 (1969).

[32] A. J. Leggett, in Modern Trends in the Theory of Condensed Matter, edited by A. Pekalski and J. Przystawa, Lecture Notes in Physics (Springer-Verlag, Berlin, 1980), Vol. 115, p. 13.

[33] P. Nozieres and S. Schmitt-Rink, J. Low Temp. Phys. 59, 195 (1985).

[34] N. Andrenacci, A. Perali, P. Pieri, and G. C. Strinati, Phys. Rev. B 60, 12410 (1999).

[35] B. O. Kerbikov, Surv. High Energy Phys. 20, 47 (2006).
[36] B. O. Kerbikov, Phys. At. Nucl. 65, 1918 (2002).

[37] A. I. Larkin and A. A. Varlamov, Theory of Fluctuations in Superconductors (Clarendon Press, Oxford, 2005).

[38] A. P. Levanyuk, Sov. Phys. JETP 9, 571 (1959).

[39] V. L. Ginzburg, Sov. Solid State Phys. 2, 61 (1960).

[40] B. O. Kerbikov, Phys. At. Nucl. 68, 890 (2005).

[41] B. O. Kerbikov and M. A. Andreichikov, Phys. Rev. D 91, 074010 (2015).

[42] L. D. Landau and I. M. Khalatnikov, Dokl. Akad. Nauk USSR 96, 469 (1954).

[43] L. P. Gor'kov and G. M. Eliashberg, Sov. Phys. JETP 27, 328 (1968).

[44] Seung-il Nam, Phys. Rev. D 86, 033014 (2012).

[45] L. D. Landau and E. M. Lifshitz, Statistical Physics Part 1, 3rd ed. (Butterworth-Heinemann, Oxford, 2005).

[46] D. E. Kharzeev, L. D. McLerran, and H. J. Warringa, Nucl. Phys. A803, 227 (2008).

[47] L. G. Aslamazov and A. I. Larkin, Sov. Phys. Solid State 10, 875 (1968).

[48] K. Maki, Prog. Theor. Phys. 40, 193 (1968).

[49] R. S. Thompson, Phys. Rev. B 1, 327 (1970).

[50] B. N. Narozhny, Sov. Phys. JETP 77, 301 (1993).

[51] L. G. Aslamazov and A. A. Varlamov, Sov. Phys. JETP 50, 1164 (1979).

[52] R. E. Glover, Phys. Lett. A 25A, 542 (1967).

[53] G. M. Eliashberg, Sov. Phys. JETP 14, 886 (1961).

[54] J. Kapusta, P. Lichard, and D. Seibert, Phys. Rev. D 44, 2774 (1991).

[55] H.-T. Ding, Nucl. Phys. A932, 500 (2014).

[56] H.-T. Ding, O. Kaczmarek, and F. Meyer, Phys. Rev. D 94, 034504 (2016).

[57] A. A. Abrikosov, L. P. Gor'kov, and I. E. Dzyaloshinskii, Methods of the Quantum Field Theory in Statistical Physics (Dover, New York, 1963).

[58] B. O. Kerbikov, 2018 QCD and high energy interactions, in Proceeding of the 53th Rencontres de Moriond QCD, ARISF (2018), p. 291.

[59] B. O. Kerbikov and M. S. Lukashov, Mod. Phys. Lett. A 31, 1650179 (2016).

[60] A. Onuki, Phys. Rev. E 55, 403 (1997).

[61] G. D. Moore and O. Saremi, J. High Energy Phys. 09 (2008) 015 .

[62] N. G. Antoniou, F. K. Diakonos, and A. S. Kapoyannis, Phys. Rev. C 96, 055207 (2017).

[63] A. Monnai, S. Mukherjee, and Yi Yin, Phys. Rev. C 95, 034902 (2017). 